\title{
Inverse Scattering Problem with Underdetermined Data
}

\author{
A.G. Ramm* \\ Mathematics Department, Kansas State University, Manhattan, KS 66506-2602, USA
}

\begin{abstract}
Consider the Schrödinger operator $-\nabla^{2}+q$ with a smooth compactly supported potential $q, q=q(x), x \in \mathbf{R}^{3}$.

Let $A(\beta, \alpha, k)$ be the corresponding scattering amplitude, $k^{2}$ be the energy, $\alpha \in S^{2}$ be the incident direction, $\beta \in S^{2}$ be the direction of scattered wave, $S^{2}$ be the unit sphere in $\mathbf{R}^{3}$. Assume that $k=k_{0}>0$ is fixed, and $\alpha=\alpha_{0}$ is fixed. Then the scattering data are $A(\beta)=$ $A\left(\beta, \alpha_{0}, k_{0}\right)=A_{q}(\beta)$ is a function on $S^{2}$. The following inverse scattering problem is studied: IP: Given an arbitrary $f \in L^{2}\left(S^{2}\right)$ and an arbitrary small number $\epsilon>0$, can one find $q \epsilon$ $C_{0}^{\infty}(D)$, where $D \in \mathbf{R}^{3}$ is an arbitrary fixed domain, such that $\left\|A_{q}(\beta)-f(\beta)\right\|_{L^{2}\left(S^{2}\right)}<\epsilon$ ?

A positive answer to this question is given. A method for constructing such a $q$ is proposed. There are infinitely many such $q$, not necessarily real-valued.
\end{abstract}

Keywords and phrases: underdetermined data, inverse scattering, fixed energy, fixed incident direction

Mathematics Subject Classification: 35R30, 35J10, 81Q05

\section{Introduction}

Consider the scattering problem:

Find the solution to the equation

$$
\left[\nabla^{2}+k^{2}-q(x)\right] u=0 \quad \text { in } \mathbf{R}^{3}
$$

such that

$$
u=e^{i k \alpha \cdot x}+A(\beta, \alpha, k) \frac{e^{i k r}}{r}+o\left(\frac{1}{r}\right), \quad r=|x| \rightarrow \infty, \quad \frac{x}{r}=\beta,
$$

where $\alpha \in S^{2}$ is a given unit vector, $S^{2}$ is the unit sphere, $k=$ const $>0, k^{2}$ is the energy, $\alpha$ is the direction of the incident plane wave $u_{0}:=e^{i k \alpha \cdot x}$ is the incident plane wave, $\beta$ is the direction of the scattered wave. The function

$$
A(\beta, \alpha, k)=A_{q}(\beta, \alpha, k)
$$

is called the scattering amplitude corresponding to the potential $q(x)$.

*Corresponding author. E-mail: ramm@math.ksu.edu 
If $q(x) \in C_{0}^{\infty}\left(\mathbf{R}^{3}\right)$ and is a real valued function, then the scattering problem (1.1)-(1.2) has a unique solution, the scattering solution. There is a large literature on this topic, see, for example, [1], [2], [3], and and references therein. The scattering theory has been developed for much larger classes of potentials, not necessarily smooth and compactly supported.

We prove existence and uniqueness of the scattering solution assuming that $\operatorname{Im} q \leq 0$, see Lemma 2.5 in Section 2. The inverse scattering problem consists in finding $q(x)$ in a certain class of potentials from the knowledge of the scattering data $A(\beta, \alpha, k)$ on some subsets of the set $S^{2} \times S^{2} \times \mathbf{R}_{+}$, where $\mathbf{R}_{+}=[0, \infty)$. If $A(\beta, \alpha, k)$ is known everywhere in the above set, then the inverse scattering problem is easily seen to be uniquely solvable in the class of $L_{0}^{2}\left(\mathbf{R}^{3}\right)$, that is, in the class of compactly supported square-integrable potentials, and in much larger class of potentials. If the scattering data is given at a fixed energy, and $k=k_{0}>0$ for all $\beta \in S^{2}$ and all $\alpha \in S^{2}$, then uniqueness of the solution to inverse scattering problem was proved originally in [4]. An algorithm for finding $q(x)$ from the exact fixed-energy scattering data and from noisy fixed-energy scattering data was given in [5], where the error estimates of the proposed solution were also obtained, see Chapter 5 in [6].

Only recently the uniqueness of the solution to inverse scattering problem with non-over-determined data $A(-\beta, \beta, k)$ and $A\left(\beta, \alpha_{0}, k\right)$ was proved, see [7], and [8], [12].

The data $A(-\beta, \beta, k), \forall \beta \in S^{2}$ and all $k>0$ are the back-scattering data, the $A\left(\beta, \alpha_{0}, k\right), \forall \beta \in S^{2}$ and $\forall k>0$ are the fixed incident direction data. The scattering data are called "non-over-determined" if these data depend on the same number of variables as the unknown potential, that is on three variables in the above problems.

Note that the data $A\left(\beta, \alpha, k_{0}\right), \forall \alpha, \beta \in S^{2}$ and a fixed $k=k_{0}>0$ are over-determined: they depend on four variables while $q$ depends on three variables.

The inverse problem IP with the data $A(\beta):=A_{q}(\beta):=A\left(\beta, \alpha_{0}, k_{0}\right)$ is under-determined: its data depend on two variables. This problem, in general, does not have a unique solution in sharp contrast to the inverse scattering problems mentioned above. The IP was not studied in the literature, except in the paper [10]. The proofs in the current paper are more detailed, the potential is allowed to be complex-valued with $\operatorname{Im} q \leq 0$, and the uniqueness of the scattering solution is proved for this class of the potentials.

In this paper the IP is studied further. Assume that $D \subset \mathbf{R}^{3}$ is an arbitrary fixed bounded domain.

Let us formulate the inverse problem:

IP: Given an arbitrary $f \in L^{2}\left(S^{2}\right)$ and an arbitrary small number $\epsilon>0$, find a $q \in C_{0}^{\infty}(D)$ such that

$$
\|A(\beta)-f(\beta)\|_{L^{2}\left(S^{2}\right)}<\epsilon .
$$

The IP's formulation differs from the formulation of the inverse scattering problems discussed earlier:

i) There is no unique solution for the problem we are discussing,

ii) In place of the exact (or noisy) scattering data a function $f(\beta)$ on $S^{2}$ is given, which, in general, is not a scattering amplitude at a fixed $\alpha=\alpha_{0}$ and a fixed $k=k_{0}$ corresponding to any potential from $L^{2}(D)$.

The main results of this paper include:

a) A proof of the existence of $q \in C_{0}^{\infty}(D)$ such that (1.4) holds;

b) A method for finding a potential $q \in C_{0}^{\infty}(D)$ for which (1.4) holds;

c) An analytic formula for a function $h=q u$, where $u$ is the scattering solution at a fixed $k$ and a fixed $\alpha$, corresponding to $q$.

In section 2 we prove that the set $\{A(\beta)\}$ corresponding to all $q \in C_{0}^{\infty}(D)$, is dense in $L^{2}\left(S^{2}\right)$, and that the set of functions $\{h\}=\{q u\}$ is dense in $L^{2}(D)$ when $q$ runs through all of $L^{2}(D)$. Here $u=u(x)=u\left(x, \alpha_{0}, k_{0}\right)$ is the scattering solution corresponding to the potential $q$, that is, the solution to the scattering problem (1.1)-(1.2) with $\alpha=\alpha_{0} \in S^{2}$ and $k=k_{0}>0$.

In section 3 an analytical formula for $q$ is given. The $q$ computed by this formula generates $A(\beta)$ satisfying (1.4). 
We do not discuss in this paper the relation of our results with the theory of creating materials with a desired refraction coefficient, see [9], [11], [14].

\section{The density of the set $A(\beta)$ in $L^{2}\left(S^{2}\right)$}

This Section is the main one. The logic of the proof of the main result, namely, the validity of formula (1.4), can be described as follows.

First, it is proved that the set of functions $\left\{-\frac{1}{4 \pi} \int_{D} e^{-i k \beta \cdot y} h(y) d y\right\}$ is dense in $L^{2}\left(S^{2}\right)$ when $h$ runs through $L^{2}(D)$, see Theorem 2.2 .

Then we prove that the set $\{q u\}$ is dense in $L^{2}(D)$ when $q$ runs through $L^{2}(D)$ and $u$ is the scattering solution corresponding to $q$, see Theorem 2.4.

Combining these two results one gets the desired inequality (1.4).

Let us start by proving the following lemma.

Lemma 2.1. If the set $\{A(\beta)\}$ is dense in $L^{2}\left(S^{2}\right)$ when $q$ runs through all of $L^{2}(D)$, then it is dense in $L^{2}\left(S^{2}\right)$ when $q$ runs through $C_{0}^{\infty}(D)$.

Proof. The set $C_{0}^{\infty}(D)$ is dense in $L^{2}(D)$ (in $L^{2}(D)$ norm), and the scattering amplitude $A(\beta)$ depends continuously in the norm of $L^{2}\left(S^{2}\right)$ on $q$, that is,

$$
\left\|A_{q_{1}}(\beta)-A_{q_{2}}(\beta)\right\|_{L^{2}\left(S^{2}\right)} \leq c\left\|q_{1}-q_{2}\right\|_{L^{2}(D)} .
$$

Estimate (2.1) follows, for example, from the known lemma of the author (see [6], p.262):

$$
-4 \pi\left[A_{q_{1}}(\beta)-A_{q_{2}}(\beta)\right]=\int_{D}\left[q_{1}(x)-q_{2}(x)\right] u_{1}\left(x, \alpha_{0}, k_{0}\right) u_{2}\left(x,-\beta, k_{0}\right) d x,
$$

and the well-known estimate

$$
\sup _{x \in D}|u(x, \alpha, k)| \leq c
$$

where $c>0$ is a constant depending on the $L^{2}(D)$ norm of $q$ and uniform with respect to $k \in[a, \infty)$, $a>0$ is a constant and $\alpha \in S^{2}$. Lemma 2.1. is proved.

Thus, in what follows it is sufficient to establish the density of the set $\left\{A_{q}(\beta)\right\}$ in $L^{2}\left(S^{2}\right)$ when $q$ runs through $L^{2}(D)$.

Theorem 2.2. For any $f \in L^{2}\left(S^{2}\right)$ and any $\epsilon>0$ there exists a $q \in C_{0}^{\infty}(D)$ such that estimate (1.4) holds, where $A(\beta)=A_{q}(\beta)$ is the scattering amplitude corresponding to $q$, and $\alpha=\alpha_{0} \in S^{2}, k=k_{0}>0$ are fixed.

Proof. By Lemma 2.1, it is sufficient to prove that the set $\{A(\beta)\}$ is dense in $L^{2}\left(S^{2}\right)$ when $q$ runs through all of $L^{2}(D)$. Assuming the contrary, one finds a function $f \in L^{2}\left(S^{2}\right)$ which is orthogonal in $L^{2}\left(S^{2}\right)$ to any function $A(\beta)$. It is well-known that

$$
-4 \pi A(\beta)=\int_{D} e^{-i k \beta \cdot y} h(y) d y, \quad h(y):=q(y) u(y),
$$

where $u(y)=u\left(y, \alpha_{0}, k_{0}\right)$ is the scattering solution. Using the orthogonality of $f$ to $A(\beta)$, one gets

$$
\int_{S^{2}} f(\beta) \int_{D} e^{-i k \beta \cdot y} h(y) d y d \beta=0, \quad \forall q \in L^{2}(D) .
$$

We prove later, see Theorem 2.4, that when $q$ runs through all of $L^{2}(D)$, the corresponding $h$ runs through a set $\tilde{L}$ dense in $L^{2}(D)$. Taking this for granted, one can replace in $(2.5)$ the expression $\forall q \in L^{2}(D)$ by $\forall h \in L^{2}(D)$, and rewrite (2.5) as follows:

$$
\int_{D} h(y) \int_{S^{2}} f(\beta) e^{-i k \beta \cdot y} d \beta d y=0, \quad \forall h \in \tilde{L} .
$$


This implies

$$
\int_{S^{2}} f(\beta) e^{-i k \beta \cdot y} d \beta=0, \quad \forall y \in D,
$$

where $k=k_{0}$ is fixed. In what follows, we write everywhere $k$ for $k_{0}$ and $\alpha$ for $\alpha_{0}$. The integral in (2.7) can be considered as the Fourier transform of a compactly supported distribution

$$
g(\lambda, \beta):=g(\xi):=f\left(\frac{\xi}{|\xi|}\right) \frac{\delta(|\xi|-k)}{|\xi|^{2}}
$$

where $\xi \in \mathbf{R}^{3}, \lambda=|\xi|, \beta=\frac{\xi}{\lambda}$, and

$$
\tilde{g}(y):=\int_{\mathbf{R}^{3}} g(\xi) e^{-i \xi \cdot y} d \xi=\int_{0}^{\infty} \lambda^{2} d \lambda \int_{S^{2}} e^{-i \lambda \beta \cdot y} g(\lambda, \beta) d \beta .
$$

Since distribution (2.8) is supported on the sphere $|\xi|=k$, which is a compact set in $\mathbf{R}^{3}$, its Fourier transform is an entire function of $y$. This function vanishes in an open in $\mathbf{R}^{3}$ set $D$ by (2.7). Therefore, it vanishes everywhere in $\mathbf{R}^{3}$. By the injectivity of the Fourier transform one concludes that $f(\beta)=0$. Therefore the assumption that the set $\{A(\beta)\}$ is not dense in $L^{2}\left(S^{2}\right)$ is false. Theorem 2.2 is proved under the assumption that the set $\{h\}$ is dense in $L^{2}(D)$ when $q$ runs through all of $L^{2}(D)$. In Theorem 2.4 , see below, this density statement is proved. Thus, one can consider Theorem 2.2 proved.

Remark 2.3. If one defines $A(\beta)=-\frac{1}{4 \pi} \int_{D} e^{-i k \beta \cdot y} h(y) d y$ and assumes that $\{h\}$ runs through a dense subset of $L^{2}(D)$, then the corresponding set $\{A(\beta)\}$ is dense in $L^{2}\left(S^{2}\right)$, as follows from our proof of Theorem 2.2.

Theorem 2.4. The set $\{h\}$ is dense in $L^{2}(D)$ when $q$ runs through a dense subset of $L^{2}(D)$, where $h(x)=q(x) u(x)$, and $u(x)$ is the scattering solution corresponding to $q=q(x)$.

Proof. If $u$ is the scattering solution, then

$$
\begin{aligned}
& u(x)=u_{0}(x)-\int_{D} g(x, y) h(y) d y, x \in \mathbf{R}^{3}, \quad u_{0}(x):=e^{i k \alpha \cdot x}, \quad h=q u, \\
& g(x, y)=\frac{e^{i k|x-y|}}{4 \pi|x-y|}, \quad x, y \in \mathbf{R}^{3} .
\end{aligned}
$$

Define

$$
q(x)=\frac{h(x)}{u(x)}=\frac{h(x)}{u_{0}(x)-\int_{D} g(x, y) h(y) d y}, \quad x \in \mathbf{R}^{3} .
$$

If the function $q(x)$, defined in (2.12), belongs to $L^{2}(D)$, then the function $u(x)$, defined in (2.10), is the scattering solution, corresponding to $q \in L^{2}(D)$, defined by formula (2.12). Uniqueness of the scattering solution is guaranteed if $\operatorname{Im} q \leq 0$ by the following Lemma 2.5 .

Lemma 2.5. Assume that $\operatorname{Im} q \leq 0$ and $q \in L^{2}(D), q=0$ in $D:=\mathbf{R}^{3} \backslash D$. Then there exists a unique scattering solution, that is, the solution to problem (1.1)-(1.2). This solution is also the unique solution to equation (2.10) if $h=q u$.

Proof. It is sufficient to prove uniqueness of the scattering solution. Indeed, the scattering solution solves a Fredholm-type Lippmann-Schwinger equation,

$$
u(x)=u_{0}(x)-\int_{D} g(x, y) q(y) u(y) d y,
$$

and the uniqueness of the solution to this equation implies the existence of this solution by the Fredholm alternative. 
Suppose that there are two scattering solutions, $u_{1}$ and $u_{2}$, that is, solution to problem (1.1)-(1.2) (with $\alpha=\alpha_{0}$ and $k=k_{0}>0$ ). Then the function $v:=u_{1}-u_{2}$ solves equation (1.1) and satisfies the radiation condition at infinity:

$$
\begin{aligned}
& {\left[\nabla^{2}+k^{2}-q(x)\right] v=0 \quad \text { in } \mathbf{R}^{3},} \\
& \frac{\partial v}{\partial r}-i k v=o\left(\frac{1}{r}\right), \quad r:=|x| \rightarrow \infty .
\end{aligned}
$$

Multiply equation (2.13) by $\bar{v}$, the bar stands for complex conjugate, and the complex conjugate of (2.13) by $v$ and subtract from the first equation the second. The result is

$$
\bar{v}\left(\nabla^{2}+k^{2}\right) v-v\left(\nabla^{2}+k^{2}\right) \bar{v}-(q-\bar{q})|v|^{2}=0
$$

Integrate (2.15) over a ball $B_{R}$ of large radius $R$, centered at the origin, and use the Green's formula to get

$$
\int_{|x|=R}\left(\bar{v} \frac{\partial v}{\partial r}-v \frac{\partial \bar{v}}{\partial r}\right) d s-2 i \int_{B_{R}} \operatorname{Im} q(x)|v(x)|^{2} d x=0 .
$$

Using the radiation condition (2.14) one rewrites (2.16) as

$$
2 i k \int_{|x|=R}|v|^{2} d s-2 i \int_{B_{R}} \operatorname{Im} q|v|^{2} d x+o(1)=0,
$$

where $o(1) \rightarrow 0$ as $R \rightarrow \infty$. Thus, if $\operatorname{Im} q \leq 0$ relation (2.17) implies that

$$
\lim _{R \rightarrow \infty} \int_{|x|=R}|v|^{2} d s=0
$$

Condition (2.18) and equation (2.13) with a compactly supported $q \in L^{2}(D)$ imply that $v=0$ in $\mathbf{R}^{3}$, see Lemma 2.1 on p.25 in [13].

The scattering solution solves equation (2.10), because it satisfies the equation

$$
u=u_{0}-\int_{D} g(x, y) q(y) u(y) d y
$$

so that with the notation $h:=q u$ one gets from (2.19) equation (2.10).

Conversely, assume that $u$ is defined by equation (2.10) and $h$ in (2.10) is equal to $q u$, where $q$ is defined by formula (2.12) and $q \in L^{2}(D)$. Then this $u$ solves equation (2.19), and, therefore, it satisfies equations (1.1) and (1.2). To check this, apply the operator $\nabla^{2}+k^{2}$ to equation (2.10) and use the known formula $\left(\nabla^{2}+k^{2}\right) g=-\delta(x-y)$.

The result is $\left(\nabla^{2}+k^{2}\right) u=h=q u$, so equation (1.1) holds. The radiation condition holds because $q$ is compactly supported and $g$ satisfies the radiation condition. Lemma 2.5 is proved.

Although the scattering solution, in general, is not unique if $\operatorname{Im} q>0$ and may not exist in this case, it does exist, even if $\operatorname{Im} q>0$, if $q$, defined by formula (2.12), belongs to $L^{2}(D)$. Indeed, then $q u=h$ and

$$
u(x)=u_{0}(x)-\int_{D} g(x, y) q(y) u(y) d y,
$$

so that $u:=u_{0}(x)-\int_{D} g(x, y) h(y) d y$ is the scattering solution.

Let us assume now that the function (2.12) does not belong to $L^{2}(D)$. Since $h \in L^{2}(D)$ the function $u(x) \in H_{l o c}^{2}\left(\mathbf{R}^{3}\right)$, where $H_{l o c}^{2}\left(\mathbf{R}^{3}\right)$ is the Sobolev space.

Changing $h$ slightly one may assume that $h$ is a smooth bounded function in $D$. Such a change leads to a small change of the scattering data $A(\beta)$ in $L^{2}\left(S^{2}\right)$ norm. Thus, we will assume below that $h$ is bounded 
in $D$ in absolute value. The function (2.12) does not belong to $L^{2}(D)$ if and only if the denominator in (2.12) has zeros. Let

$$
N:=N(u):=\{x: u(x)=0, x \in D\}, \quad N_{\delta}:=\{x:|u(x)|<\delta, x \in D\},
$$

where $\delta>0$ is a small number, and let $D_{\delta}:=D \backslash N_{\delta}$.

The idea of the argument below is to show that the set $N$ is generically a line in $\mathbf{R}^{3}$, and that there exists a function

$$
h_{\delta}(x)= \begin{cases}h(x) & \text { in } D_{\delta} \\ 0 & \text { in } N_{\delta}\end{cases}
$$

such that

$$
\left\|h_{\delta}-h\right\|_{L^{2}(D)} \leq c \delta^{2} .
$$

Moreover, the function $h_{\delta} \in L^{\infty}(D)$. This function can be made smooth by an approximation by a $C_{0}^{\infty}(D)$-function. The corresponding smooth $h_{\delta}$ generates $A_{\delta}(\beta)$ which differs slightly from the original $f(\beta)$. The corresponding $u_{\delta}(x)$ can be defined as follows:

$$
u_{\delta}(x):=u_{0}(x)-\int_{D} g(x, y) h_{\delta}(y) d y, \quad x \in \mathbf{R}^{3},
$$

where

$$
q_{\delta}:=\left\{\begin{array}{ll}
\frac{h_{\delta}}{u_{\delta}} & \text { in } D_{\delta}, \\
0 & \text { in } N_{\delta},
\end{array} \quad q_{\delta} \in L^{\infty}(D) .\right.
$$

Consequently, we will prove that a small change of $h$ may be arranged in such a way that the corresponding change of $q$ leads to a potential $q_{\delta}$ which belongs to $L^{\infty}(D)$.

Lemma 2.6. The set $N$ is a line in $\mathbf{R}^{3}$.

Proof. Let $u=u_{1}+i u_{2}$, where $u_{1}=\Re u$ and $u_{2}=\Im u$. Then the set $N$ is defined by two equations in $\mathbf{R}^{3}$ :

$$
u_{1}(x)=0, \quad u_{2}(x)=0, \quad x \in \mathbf{R}^{3} .
$$

The functions $u_{j} \in H_{l o c}^{2}\left(\mathbf{R}^{3}\right), j=1,2$, because $u \in H_{l o c}^{2}\left(\mathbf{R}^{3}\right)$. Therefore, each of the two equations in (2.24) is an equation of a surface.

The two simultaneous equations (2.24) generically describe a line $\ell$ in $\mathbf{R}^{3}$. By a small perturbation of $h$ one may ensure that the line $\ell:=\left\{x: u_{1}(x)=0, u_{1}(x)=0, x \in D\right\}$ is smooth in $D$ and the vectors $\nabla u_{j}, j=1,2$, on $\ell$ are linearly independent.

Lemma 2.6 is proved.

Lemma 2.7. There exists a function (2.20) such that (2.21) holds.

Proof. Consider a tubular neighborhood of the line $\ell$ in $D$. This neighborhood is described by the inequality $\rho(x, \ell) \leq \delta$, where $\rho(x, \ell)$ is the distance between $x$ and $\ell$. Choose the origin $O$ on $\ell$ and let the coordinates $s_{j}, j=1,2$, in the plane orthogonal to $\ell$, be directed along the vectors $\left.\nabla u_{j}\right|_{l}$ respectively, while the third coordinate $s_{3}$ be directed along the tangent line to $\ell$. The Jacobian $J$ of the transformation $\left(x_{1}, x_{2}, x_{3}\right) \rightarrow\left(s_{1}, s_{2}, s_{3}\right)$ is non-singular, $|J|+\left|J^{-1}\right| \leq c$, because $\nabla u_{j}, j=1,2$ are linearly independent.

Define $h_{\delta}$ by formula $(2.20), u_{\delta}$ by formula (2.22), and $q_{\delta}$ by formula (2.23).

Note that

$$
u_{\delta}(x)=u(x)+\int_{D} g(x, y)\left[h(y)-h_{\delta}(y)\right] d y .
$$

Since $h_{\delta}=h$ in $D_{\delta}$ and $h_{\delta}=0$ in $N_{\delta}$, one obtains inequality (2.21):

$$
\left\|h_{\delta}-h\right\|_{L^{2}(D)}=\|h\|_{L^{2}\left(N_{\delta}\right)} \leq c \delta^{2},
$$


because $h$ is bounded and $\delta^{2}$ is proportional to the area of the cross section of the tubular neighborhood. Furthermore,

$$
\left|u_{\delta}(x)\right| \geq|u(x)|-c \int_{N_{\delta}} \frac{d y}{4 \pi|x-y|}, \quad c=\max _{x \in N_{\delta}}|h(x)| .
$$

Denote

$$
I(\delta):=\sup _{x \in D_{\delta}} \int_{N_{\delta}} \frac{d y}{4 \pi|x-y|}
$$

By construction

$$
|u(x)| \geq \delta \quad \text { if } x \in D_{\delta} .
$$

Therefore, inequality (2.26) implies

$$
\left|u_{\delta}(x)\right| \geq \delta-c I(\delta) \quad \forall x \in D_{\delta} .
$$

Let us estimate $I_{\delta}$ as $\delta \rightarrow 0$ with the aim to prove that

$$
\inf _{x \in D_{\delta}}\left|u_{\delta}(x)\right| \geq b(\delta)>0, \quad \lim _{\delta \rightarrow 0} \frac{b(\delta)}{\delta}=1 .
$$

So, for sufficiently small $\delta>0$ one has $b(\delta) \geq \frac{\delta}{2}$.

If (2.30) holds, then $\left|u_{\delta}(x)\right|$ is strictly positive in $D_{\delta}$ and, therefore, $q_{\delta}:=\frac{h_{\delta}}{u_{\delta}}$ is a bounded function in $D_{\delta}$.

Since $h_{\delta}=h$ in $D_{\delta}$, one obtains

$$
\left\|q_{\delta}-q\right\|_{L^{2}\left(D_{\delta}\right)}=\left\|\frac{h_{\delta}}{u_{\delta}}-\frac{h}{u}\right\|_{L^{2}\left(D_{\delta}\right)} \leq\|h\|_{C\left(D_{\delta}\right)}\left\|\frac{u-u_{\delta}}{u_{\delta} u}\right\|_{L^{2}\left(D_{\delta}\right)} \leq c \delta \ln \frac{1}{\delta}
$$

Here the following inequalities for $x \in D_{\delta}$ were used

$$
\begin{aligned}
& || h \|_{C\left(D_{\delta}\right)} \leq c, \quad\left|u_{\delta} u\right| \geq \frac{\delta^{2}}{2} \quad \text { in } D_{\delta} \\
& \left|u_{\delta}(x)-u(x)\right| \leq \int_{N_{\delta}}|g(x, y) h(y)| d y \leq c \delta \int_{N_{\delta}} \frac{d y}{4 \pi|x-y|} \leq c \delta I(\delta) .
\end{aligned}
$$

Here and throughout by $c>0$ various constants, independent of $\delta$, are denoted.

To estimate $I(\delta)$ one argues as follows:

$$
I(\delta) \leq \frac{1}{4 \pi} \int_{N_{\delta}} \frac{d y}{|y|} \leq c \int_{0}^{c \delta} \rho d \rho \int_{0}^{1} \frac{d s_{3}}{\sqrt{\rho^{2}+s_{3}^{2}}} \leq c \delta^{2} \ln \frac{1}{\delta},
$$

where the unit 1 is a finite coordinate along the $s_{3}$ axis and we have used the following estimate:

$$
\int_{0}^{1} \frac{d s_{3}}{\sqrt{\rho^{2}+s_{3}^{2}}}=\left.\ln \left(s_{3}+\sqrt{\rho^{2}+s_{3}^{2}}\right)\right|_{0} ^{1} \leq c \ln \frac{1}{\rho}, \quad \rho \rightarrow 0
$$

where $c>1$ is a constant. Estimates (2.29) and (2.34) imply inequality (2.31). Thus, the existence of a function (2.20) is proved, and

$$
\left\|h_{\delta}-h\right\|_{L^{2}(D)}=\|h\|_{L^{2}\left(N_{\delta}\right)} \leq c \int_{N_{\delta}} d x \leq c \delta^{2} .
$$

Lemma 2.7 is proved. 
From Lemma 2.7 the conclusion of Theorem 2.4 follows. Indeed, if $h \in L^{2}(D)$ is an arbitrary function and $q$, defined by formula (2.12), belongs to $L^{2}(D)$, then, as was proved above, the $u$, defined by formula (2.10), is the scattering solution. This scattering solution is unique if $\operatorname{Im} q \leq 0$ by Lemma 2.5. If $q$, defined by formula (2.12), does not belong to $L^{2}(D)$, then there is a bounded function $q_{\delta}(x)$, approximating $q$, see (2.31), such that the corresponding $h_{\delta}$ approximates $h(x)$ well, see $(2.35)$, and the corresponding $u_{\delta}(x)$ is the scattering solution corresponding to $q_{\delta}(x)$.

Theorem 2.4 is proved.

\section{Formulas for solving inverse scattering problem with fixed $\alpha$ and $k>0$}

The inverse problem (IP) was formulated in the Introduction. Given $\epsilon>0$ and an arbitrary $f(\beta) \in L^{2}\left(S^{2}\right)$ we first find $h(x) \in L^{2}(D)$ such that

$$
\left\|f(\beta)+\frac{1}{4 \pi} \int_{D} e^{-i k \beta \cdot y} h(y) d y\right\|_{L^{2}\left(S^{2}\right)} \leq \epsilon
$$

This can be done (non-uniquely !) in many ways. Let us describe one of the ways. Without loss of generalities assume that $D=B=B_{R}$ is a ball of radius $R$ centered at the origin. Expand the plane wave $e^{-i k \beta \cdot y}$ and $h(y)$ into the spherical harmonics series:

$$
\begin{aligned}
& e^{-i k \beta \cdot y}=\sum_{l=0}^{\infty} 4 \pi i^{l} j_{l}(k r) Y_{l}(-\beta) \overline{Y_{l}\left(y^{0}\right)}, \quad r=|y|, \quad y^{0}:=\frac{y}{r}, \\
& j_{l}(r):=\left(\frac{\pi}{2 r}\right)^{1 / 2} J_{l+\frac{1}{2}}(r), \quad \frac{4 \pi}{2 l+1} \sum_{m=-l}^{l} Y_{l m}\left(x^{0}\right) \overline{Y_{l m}\left(y^{0}\right)}=P_{l}\left(x^{0} \cdot y^{0}\right),
\end{aligned}
$$

$J_{l+\frac{1}{2}}(r)$ is the Bessel function regular at the origin, $Y_{l}(\alpha)$ are the spherical harmonics:

$$
\begin{aligned}
& Y_{l}(\alpha)=Y_{l, m}(\alpha)=\frac{(-1)^{\frac{m+|m|}{2}} i^{l}}{\sqrt{4 \pi}}\left[\frac{(2 l+1)(l-|m|) !}{(l+|m|) !}\right]^{1 / 2} e^{i m \varphi} P_{l, m}(\cos \theta), \\
& -l \leq m \leq l, \\
& P_{l, m}(\cos \theta)=(\sin \theta)^{m} \frac{d^{m} P_{l}(\cos \theta)}{(d \cos \theta)^{m}}, \quad P_{l}(t)=\frac{1}{2^{l} l !} \frac{d^{l}\left(t^{2}-1\right)^{l}}{d t^{l}},
\end{aligned}
$$

$t=\cos \theta$, the unit vector $\alpha$ is described by the spherical coordinates $(\theta, \varphi), 0 \leq \varphi<2 \pi, 0 \leq \theta \leq \pi,-l \leq$ $m \leq l$, one has

$$
Y_{l, m}(-\alpha)=(-1)^{l} Y_{l, m}(\alpha), \quad \overline{Y_{l, m}(\alpha)}=(-1)^{l+m} Y_{l,-m}(\alpha),
$$

where the overline stands for complex conjugate. The summation in (3.2) and below is understood as $\sum_{l=0}^{\infty} \sum_{m=-l}^{l}$.

Let

$$
h(y)=\sum_{l=0}^{\infty} h_{l}(r) Y_{l}\left(y^{0}\right) .
$$

It is well-known that

$$
\left(Y_{l, m} Y_{l^{\prime}, m^{\prime}}\right)_{L^{2}\left(S^{2}\right)}=\delta_{l l^{\prime}} \delta_{m m^{\prime}}
$$

where $\delta_{l l^{\prime}}$ is the Kronecker delta.

Let

$$
f_{L}(\beta):=\sum_{l=0}^{L} f_{l} Y_{l}(\beta),
$$


where $f_{l}:=\left(f, Y_{l}\right)_{L^{2}\left(S^{2}\right)}$ are the Fourier coefficients of $f$. For sufficiently large $L$ one has

$$
\left\|f-f_{L}\right\|_{L^{2}\left(S^{2}\right)}<\epsilon / 2 \text {. }
$$

Thus, if

$$
\left\|f_{L}(\beta)+\frac{1}{4 \pi} \int_{B} e^{-i k \beta \cdot y} h(y) d y\right\|_{L^{2}\left(S^{2}\right)}<\epsilon / 2,
$$

then inequality (3.1) holds. Therefore, practically it is sufficient to find $h$ satisfying inequality (3.11). Substitute (3.7) and (3.2) into the equation

$$
\int_{B} e^{-i k \beta \cdot y} h(y) d y=-4 \pi f_{L}(\beta)
$$

and use (3.8) and (3.6) to get

$$
4 \pi(-i)^{l} \int_{0}^{R} r^{2} j_{l}(k r) h_{l}(r) d r=-4 \pi f_{l}^{(L)}, \quad 0 \leq l \leq L .
$$

Equation (3.13) can be written as

$$
(-i)^{l+2} \int_{0}^{R} r^{2} j_{l}(k r) h_{l}(r) d r=f_{l}^{(L)}, \quad \forall l \geq 0 .
$$

Recall that $f_{l}^{(L)}=f_{l, m}^{(L)}$ and $h_{l}(r)=h_{l, m}(r)$.

Equation (3.14) has many solutions.

Denote by $h_{l}^{\perp}(r)$ any function such that

$$
\int_{0}^{R} r^{2} j_{l}(k r) h_{l}^{\perp}(r) d r=0 .
$$

Then the general solution to equations (3.14) has the form

$$
h_{l}(r)=(-i)^{-l-2} f_{l}^{(L)} \gamma_{l} j_{l}(k r)+c_{l} h_{l}^{\perp}(r), \quad 0 \leq l \leq L,
$$

where $c_{l}$ are arbitrarily constants, and

$$
\gamma_{l}:=\frac{1}{\int_{0}^{R} d r r^{2} j_{l}^{2}(k r)} .
$$

We have proved the following result.

Theorem 3.1. The function (3.7), with $h_{l}$ defined in (3.16) for $0 \leq l \leq L$ and $h_{l}=0$ for $l>L$, solves equation (3.12). If $L=L(\epsilon)$ is sufficiently large, so that (3.10) holds, then the function

$$
h^{(L)}(y)=\sum_{l=0}^{L} h_{l}^{(L)}(r) Y_{l}\left(y^{0}\right), \quad r=|y|, \quad y^{0}=\frac{y}{r},
$$

with $h_{l}^{(L)}(r)$ defined in (3.16), satisfies inequality (3.1).

Let us give a formula for a potential $q$ such that $h=q u$ approximates $h^{(L)}$ with a desired accuracy. This $q$ is a solution to the inverse scattering problem (IP).

Given $h^{(L)}(y)$ defined in (3.18), let us denote it $h(y)$ for simplicity. Using this function, calculate $q(x)$ formula (2.12). If this $q \in L^{2}(D)$, then the inverse problem (IP) is solved. There is no guarantee that $\operatorname{Im} q \leq 0$. 
If formula (2.12) does not yield an $L^{2}(D)$ function, then one uses $h_{\delta}(x)$ in place of $h(x)$ and, as was proved in Theorem 2.4, obtains a potential $q_{\delta}(x)$ which is a solution to (IP).

Other computational methods can be used for finding $h(y)$ given $f(\beta)$. For example, one can choose a basis $\left\{\varphi_{j}\right\}$ in $L^{2}(B), B=D$ is a ball, and look for

$$
h_{n}(x)=\sum_{j=1}^{n} c_{j}^{(n)} \varphi_{j}(x),
$$

where $c_{j}^{(n)}$ are constants to be found from the minimization problem

$$
\left\|f(\beta)-\sum_{j=1}^{n} c_{j}^{(n)} g_{j}(\beta)\right\|_{L^{2}\left(S^{2}\right)}=\min .
$$

Here

$$
g_{j}(\beta):=-\frac{1}{4 \pi} \int_{B} e^{-i k \beta \cdot y} \varphi_{j}(y) d y
$$

A necessary condition for the minimum in (3.20) is a linear algebraic system for the coefficients $c_{j}^{(n)}, 1 \leq$ $j \leq n$.

The linear minimization problem (3.20) is well-posed for a fixed $n$, because its solution is found from the solution of a finite-dimensional linear algebraic system whose matrix is non-degenerate because the system $\left\{g_{j}\right\}_{j=1}^{n}$ is linearly independent.

\section{References}

[1] S. Agmon. Spectral properties of Schrödinger operators and scattering theory. Ann. Scuola Norm. Super. Pisa, 4, (1975), 151-218.

[2] H. Cycon, R. Froese, R. Kirsch, B. Simon. Scrödinger operators. Springer-Verlag, Berlin, 1986.

[3] D. Pearson. Quantum scattering and spectral theory. Acad. Press, London, 1988.

[4] A.G. Ramm. Recovery of the potential from fixed energy scattering data. Inverse Problems, 4 (1988), 877-886.

[5] A.G. Ramm. Stability of solutions to inverse scattering problems with fixed-energy data. Milan Journ of Math., 70 (2002), 97-161.

[6] A.G. Ramm. Inverse problems. Springer, New York, 2005.

[7] A.G. Ramm. Uniqueness theorem for inverse scattering problem with non-overdetermined data. J.Phys. A, FTC, 43 (2010), 112001.

[8] A.G. Ramm. Uniqueness of the solution to inverse scattering problem with backscattering data. Eurasian Math. Journ (EMJ), 1 (2010), no. 3, 97-111.

[9] A.G. Ramm. Distribution of particles which produces a "smart" material. Jour. Stat. Phys., 127 (2007), no. 5, $915-934$.

[10] A.G. Ramm. Inverse scattering problem with data at fixed energy and fixed incident direction, Nonlinear Analysis: Theory, Methods and Applications. 69 (2008), no.4, 1478-1484.

[11] A.G. Ramm. Wave scattering by many small bodies and creating materials with a desired refraction coefficient. Afrika Matematika, 22 (2011), no. 1, 33-55.

[12] A.G. Ramm. Uniqueness of the solution to inverse scattering problem with scattering data at a fixed direction of the incident wave. J. Math. Phys., 52 (2011), 123506.

[13] A.G. Ramm. Scattering by obstacles. D.Reidel, Dordrecht, 1986.

[14] A.G.Ramm. Scattering of Acoustic and Electromagnetic Waves by Small Bodies of Arbitrary Shapes. Applications to Creating New Engineered Materials. Momentum Press, New York, 2013. 